\title{
Neuroleptic malignant syndrome: a case for electroconvulsive therapy
}

\author{
C.C. Harland, M.M. O'Leary, ${ }^{1}$ R. Winters, J. Owens, ${ }^{1}$ \\ B. Hayes and V. Melikian
}

Departments of Medicine and ${ }^{1}$ Psychiatry Dudley Road Hospital, Birmingham B18 7QR, UK.

\begin{abstract}
Summary: A case of prolonged and refractory neuroleptic malignant syndrome was treated successfully with electroconvulsive therapy. Neuroleptic malignant syndrome and its treatment are reviewed.
\end{abstract}

\section{Introduction}

Neuroleptic malignant syndrome (NMS) is a rare and life threatening side effect of antipsychotic therapy with major tranquillisers, particularly the butyrophenones and phenothiazines. ${ }^{1-8}$ Typical features include hyperthermia, extrapyramidal muscular rigidity, dysphagia, catatonia and signs of autonomic dysfunction such as labile blood pressure, tachycardia and urinary incontinence. Leucocytosis, elevated creatine kinase and abnormal liver function tests are also described. An identical syndrome may rarely be seen in lethal catatonia which is a catatonic form of schizophrenia. ${ }^{9}$ Other diagnoses to be considered include encephalitis, meningitis, tetanus, heatstroke, and malignant hyperthermia, the last of which may be linked to NMS, ${ }^{10}$ although triggered by chemically different agents such as halothane and suxamethonium. ${ }^{11}$

Usual treatment consists of neuroleptic drug withdrawal, supportive measures, and sometimes the administration of dopamine agonists and muscle relaxants. We report a patient with NMS who did not recover until electroconvulsive therapy had been given.

\section{Case report}

A 29 year old woman was admitted to a psychiatric hospital following behavioural and mood disturbance suggestive of a hypomanic illness. She was treated with oral and intramuscular haloperidol (170 $\mathrm{mg}$ total), chlorpromazine (300 $\mathrm{mg}$ total), procyclidine and diazepam over 5 days, and had been prescribed haloperidol $(5 \mathrm{mg}$ three times daily) and a single intramuscular dose of chlorp-

Correspondence: C.C. Harland, M.R.C.P., Department of Dermatology, University Hospital, Nottingham NG7 2UH, UK.

Accepted: 12 May 1989 romazine $(75 \mathrm{mg})$ by her general practitioner on the day prior to admission. Five days later she developed marked extrapyramidal rigidity, dysphagia, urinary incontinence and a fever. She was transferred to our medical ward where all psychotropic medication was stopped.

On admission the patient was mute and adopted sustained and bizarre postures characteristic of catatonia. She was dehydrated, pyrexial and mildly hypertensive with a marked tachycardia. Abnormal laboratory results included a white cell count of $13.4 \times 10^{9} / 1$ with neutrophilia, aspartate transaminase $85 \mathrm{IU} / 1$ (normal $<45$ ), creatine kinase $1,602 \mathrm{IU} / \mathrm{l}$ (normal <150). Chest radiograph, lumbar puncture and computed tomographic (CT) brain scan were normal; urine and blood cultures were sterile and there was no demonstrable myoglobinuria. Because these features followed neuroleptic administration, NMS was diagnosed.

Intravenous dantrolene sodium $(150 \mathrm{mg} /$ day $)$ and nasogastric bromocriptine $(20 \mathrm{mg} / \mathrm{day})$ were started in addition to cooling, rehydration, and subcutaneous heparin for prophylaxis of thromboembolism. Total parenteral nutrition became necessary because nasogastric feeding was poorly tolerated. She became afebrile on the tenth day on stopping bromocriptine and dantrolene there was increasing rigidity and fever. Her condition deteriorated further in spite of the reintroduction of higher doses of dantrolene sodium $(600 \mathrm{mg}$ daily) and bromocriptine (60 mg daily). Complications of the illness included aspiration pneumonia, hypotension, unexplained polyuria, cellulitis arising from an infected intravenous cannula site, urinary tract infection related to an indwelling catheter, unexplained diarrhoea, hypokalaemia, and candida septicaemia attributable to an infected intravenous central feeding line.

Electroconvulsive therapy (ECT) was considered in view of her progressive catatonia, muscular rigidity, concomitant complications as well as the failure to respond to established conventional 
therapy. Bilateral ECT, which has been used for the treatment of the lethal form of catatonia, was administered under general anaesthesia using thiopentone and altracurium (Day 47 of hospital admission). Suxamethonium, the usual muscle relaxant employed for ECT, was avoided on account of its potential for triggering malignant hyperthermia in NMS. Four ECT treatments were delivered over 12 days. After the first treatment there was a dramatic reduction in muscular rigidity and catatonia; by the twelfth day the patient was eating and talking. Both rigidity and catatonia had completely resolved after a further four treatments over 4 weeks, the twelfth week of hospital admission, by which time the patient was walking. At this stage it seemed likely that the patient had made a full recovery from NMS. However, she did not remain afebrile until day 92 when she had received a two week course of oral flucytosine and intravenous amphotericin for persistent candida septicaemia which had been confirmed on further blood cultures. She was discharged from hospital on day 135 .

\section{Discussion}

Signs of NMS usually develop over 24-72 hours and last about 10 days after cessation of oral neuroleptics, but may last three times longer following intramuscular injections. ${ }^{4}$ Overall mortality is estimated at $20 \%$, the commonest cause of death being respiratory failure due both to decrease in chest wall compliance and to aspiration pneumonia. Other causes include cardiovascular collapse due to dehydration and hypotension, pulmonary embolism, septicaemia, and acute renal failure due to myoglobin release from damaged muscle..$^{1-4}$ Our case is unusual in that the patient remained symptomatic for at least 12 weeks following the administration of oral and intramuscular chlopromazine and haloperidol and yet survived.

The pathogenesis of NMS is uncertain but excessive dopamine-receptor blockade in the basal ganglia and hypothalamus is believed to account for the clinical features. ${ }^{1-8}$ This might explain the efficacy of dopaminergic drugs in its treatment, for example, bromocriptine and laevodopa. Dantrolene sodium dissociates excitation-contraction coupling in skeletal muscle, thereby reducing heat production and reducing contractility caused by excessive muscle activity, suggesting that the hyperthermia of NMS is, in part, due to muscle overactivity. ${ }^{1-3}$ The combination of bromocriptine and dantrolene sodium is presently advocated in the treatment of resistant cases. ${ }^{1-7}$ However, the mainstay of treatment consists of withdrawal of the neuroleptic drugs, general supportive measures, and the treatment of complications as they arise. ${ }^{1-8}$

There is limited knowledge about the use of ECT in the treatment of NMS, although it is the treatment of choice for lethal catatonia which has clinical features indistinguishable from NMS.${ }^{19} \mathrm{It}$ is ignored as potential therapy in major reviews of NMS ${ }^{1-8}$ yet 15 cases of NMS have responded to ECT. ${ }^{12-13}$ This therapeutic effect of ECT might result from increased sensitivity of dopamine receptors which has been demonstrated in animals. ${ }^{14}$ However, cardiac arrhythmias, malignant hyperthermia and hyperkalemia are potential risks of treatment. Arrhythmias have been recorded in four NMS patients thus treated, ${ }^{12}$ one of whom sustained ventricular fibrillation and coma. Malignant hyperthermia, on the other hand, has not been a reported complication of general anaesthesia despite evidence that skeletal muscle of NMS patients has a susceptibility to malignant hyperthermia when exposed to halothane in vitro. ${ }^{11}$ Life threatening hyperkalaemia has developed in a case of NMS complicated by massive rhabdomyolysis after the administration of intravenous suxamethonium to facilitate endotracheal intubation. ${ }^{15}$

There are no clear indications for using ECT in this condition, but our experience with this patient suggests a rôle when supportive measures and conventional drug therapy fail. The risks of using ECT in the treatment of NMS might be minimized by avoiding halothane and suxamethonium, and by its use in advance of impending complications. We believe that this case represents the longest recorded case of NMS to have survived.

\section{References}

I. Gibb, W.R.G., \& Lees, A.J. The neuroleptic malignant syndrome-a review. $Q J$ Med 1985, 220: 421-429.

2. Szabadi, E. Neuroleptic malignant syndrome. Br Med J 1984 , 288: $1399-1400$

3. Neuroleptic malignant syndrome (Editorial). Lancet 1984, i: 545-6

4. Caroff, S.N. The neuroleptic malignant syndrome. J Clin Psychiatry 1980, 41: 79-83.

5. Knezevic, W., Mastaglia, F.L., Lefroy, R.B. \& Fisher, A Neuroleptic malignant syndrome. Med J Aust 1984, 140: $28-30$.

6. Allsop, P. \& Twigley, A.J. The neuroleptic malignant syndrome. Case with a review of the literature. Anaesthesia 1987 , 42: $49-53$.

7. Abbott, R.J., \& Loizou, L.A. Neuroleptic malignant syndrome. Br J Psychiatry 1986, 148: 47-51.

8. Kellam, A.M.P. The neuroleptic malignant syndrome, socalled . A survey of the world literature. Br J Psychiatry 1987 , 150: $752-759$.

9. Mann, S.C., Caroff, S.N., Bleier, H.R., Wetz, W.K.R., Kling, M.A. \& Hayashida, M. Lethal catatonia. Am J Psychiatry 1986, 143: 1374-1381. 
10. Caroff, S.N., Rosenberg, H. \& Gerber, J.C. Neuroleptic malignant syndrome and malignant hyperthermia. Lancet 1983, i: 244.

11. Gronert, G.A. Malignant hyperthermia. Anesthesiology 1980, 53: 395-423.

12. Addonizio, G. \& Suzman, V.L. ECT as a treatment alternative for patients with symptoms of neuroleptic malignant syndrome. J Clin Psychiatry 1987, 48: 102-105.

13. Hermesh, H., Aizenberg, D. \& Weizman, A. A successful electroconvulsive treatment of neuroleptic malignant syndrome. Acta Psychiatr Scand 1987, 75: 237-239.
14. Willosz, M. Increased sensitivity to dopaminergic agonists after repeated electroconvulsive shock (E.C.S.) in rats. Neuropharmacology 1981, 20: 941-945.

15. George, A.L. \& Wood, C.A. Succinylcholine-induced hyperkalaemia complicating the neuroleptic malignant syndrome. Ann Int Med 1987, 106: 172. 